\title{
3-winding flyback transformer model extraction using time domain system identification
}

\author{
Tue T. Vu*, Seamus O’Driscoll ${ }^{\dagger}$ and John V. Ringwood* \\ *Department of Electronic Engineering, \\ National University of Ireland, Maynooth, Ireland \\ *Email: ttrongvu@eeng.nuim.ie, john.ringwood@eeng.nuim.ie \\ ${ }^{\dagger}$ Texas Instrument Limited, Cork, Ireland \\ †Email: seamusodriscoll@ti.com
}

\begin{abstract}
For low frequency applications, transformer model extraction has been widely investigated using both time and frequency domain data. However, the studies for high frequency transformers have been carried out in the frequency domain only. The reason is due to the complications in acquiring time domain data for high frequency transformer model estimation. This paper presents a methodology to deal with the numerical difficulties associated with time domain data collection, and to obtain a frequency-dependent model of a 3-winding flyback transformer using time domain system identification techniques. The obtained transformer model is experimentally verified.
\end{abstract}

Index Terms-Flyback transformers, frequency-dependent model, time domain, system identification.

\section{INTRODUCTION}

Given the success of the primary-side sensing approach to flyback converters operating in discontinuous conduction mode [1], the extension to continuous conduction mode (CCM) with the aim of achieving a higher performance and a lower production cost is of significant interest. However, the application of primary-side sensing to a converter in $\mathrm{CCM}$ requires an accurate model of the flyback transformer, particularly at high frequencies.

The topic of modeling and extracting models for a high frequency transformer has been investigated previously [2][5]. In general, most studies focus either on identifying a frequency-dependent winding model with an ideal core assumption using small signal excitation [4], [5], or on estimating a dynamic core loss model of a specific magnetic material using both high amplitude and high frequency voltage sources [6]. In fact, the transformer model obtained from the small signal assumption can be improved by replacing an ideal core model with a nonlinear one [7].

In this paper, we will focus on extracting the high frequency model of the flyback transformer using small signal excitation. The frequency-dependent winding model, as shown in Fig. 1, is suggested to include the core characteristic (denoted by $Z_{m}(s)$ ) and the winding configuration properties (the remainder of the model). The energy storage in the core is characterized by the magnetizing inductance

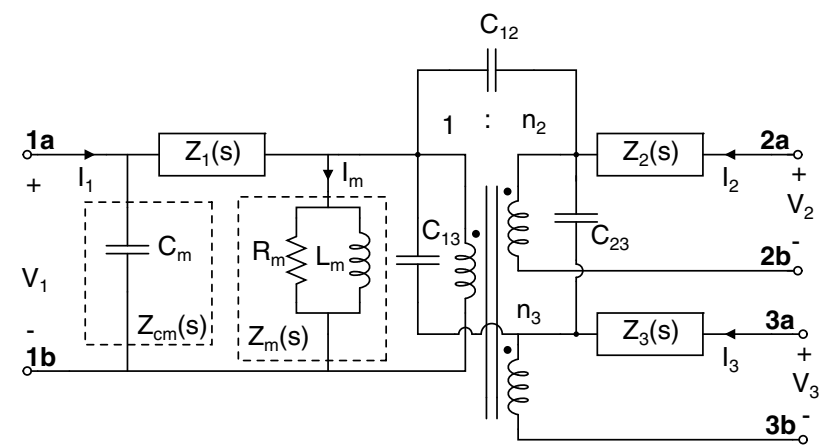

Fig. 1. Transformer winding model with a linear core.

$L_{m}$, while the resistor $R_{m}$ stands for the power losses due to the eddy current and hysteresis effect. For simplicity, the assumption was made that both $L_{m}$ and $R_{m}$ are constant with frequency. The impedance $\left\{Z_{i}(s)\right\}_{i=1}^{3}$, which represents the effect of the parasitic components in the $i$ th winding, is generally defined by,

$$
Z_{i}(s)=R_{i}(s)+s L_{i}(s), \text { for } i=1,2,3
$$

where $\left\{R_{i}(s)\right\}_{i=1}^{3}$ and $\left\{L_{i}(s)\right\}_{i=1}^{3}$ represent the power losses and the leakage inductance in the $i$ th winding, respectively. As both parameters $\left\{R_{i}(s)\right\}_{i=1}^{3}$ and $\left\{L_{i}(s)\right\}_{i=1}^{3}$ vary significantly with frequency, the winding impedance $\left\{Z_{i}(s)\right\}_{i=1}^{3}$ does not have a closed-form and depends on the configuration of each transformer. The capacitor $C_{m}$ describes the electric energy storage in each winding referred to the first winding, while the electric energy storage between windings is symbolized by $C_{12}, C_{13}$ and $C_{23}$. The transformer voltage gains $n_{2}$ and $n_{3}$ account for the coupling between transformer windings. All the capacitances $C_{m}, C_{12}, C_{13}, C_{23}$ and the voltage gains $n_{2}$, $n_{3}$ are presumed constant with frequency, in this study.

For low frequency applications, transformer model estimation has been widely studied using both time and frequency domain data [3], [8], [9]. However, the studies for high frequency transformers have been carried out in 
the frequency domain only [4], [5]. The reason behind this selection is due to the advantages of a frequency domain approach over a time domain one. Particularly, with the help of an impedance analyzer, the response of a system over a wide frequency range is much easier to collect in the frequency domain rather than in time domain. In addition, the frequency domain data, which is returned by an impedance analyzer, is also less noisy than the equivalent time domain data collected by a data acquisition card. Despite these difficulties in data collection, the time domain approach is still preferred because it requires only a simple measurement facility (such as digital oscilloscope) and provides an easy way to deal with a complex transformer model [3]. This paper presents a methodology to deal with the numerical difficulties associated with time domain data collection and to obtain a frequency-dependent model of a 3-winding flyback transformer using time domain system identification techniques. The obtained transformer model is experimentally verified.

The remainder of this paper is organized as follows; Section II introduces the continuous time system identification method, while the data collection and transformer parameter estimation are examined in Section III. The flyback transformer model is identified and verified in Section IV. Conclusions are drawn in Section V.

\section{SYSTEM IDENTIFICATION ALGORITHMS}

In general, system identification uses statistical approaches to find a mathematical model of an actual system from discrete-time input-output data $\left\{u\left(t_{k}\right), y\left(t_{k}\right)\right\}_{k=1}^{N}$. As illustrated in Fig. 2, the estimator will search for the best model parameters $\theta$ that statically minimize the error between the measured output signal $\left\{y\left(t_{k}\right)\right\}_{k=1}^{N}$ and the estimated output signal $\left\{\hat{y}\left(t_{k}\right)\right\}_{k=1}^{N}$. A model of a dynamic system can be in discrete-time or continuous-time form. A fidelity continuous-time model is of primary interest in this paper.

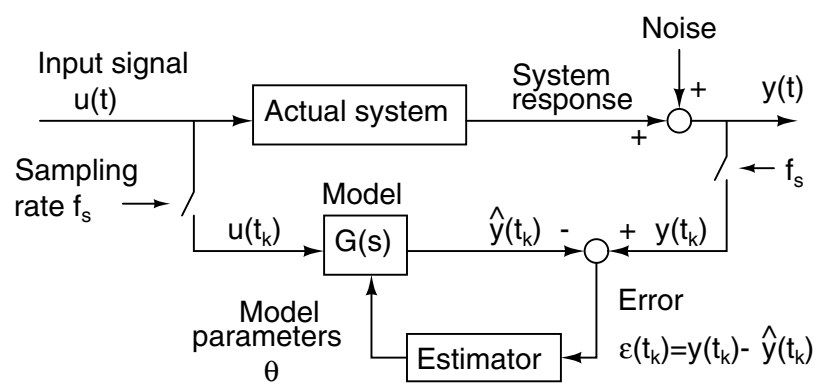

Fig. 2. A generic procedure to identify a model of an actual system from sampled data.

Various identification methods have been investigated to estimate a continuous time model $G(s)$ from sampled data [10]. In fact, we have implemented three different algorithms to obtain the winding model from sampled data. However, due to space limitation only one well known method, named Refined Simplified Instrumental Variable Method for Continuous Time System Identification (SRIVC) [11], is presented here. Let the continuous time model be

$$
G(s)=\frac{B(s)}{A(s)}=\frac{b_{1} s^{n_{b}-1}+b_{2} s^{n_{b}-2}+\ldots+b_{n_{b}}}{s^{n_{a}}+a_{1} s^{n_{a}-1}+\ldots+a_{n_{a}}} .
$$

The prediction error between the estimated and measured output data is

$$
\varepsilon(t)=y(t)-\frac{B(s)}{A(s)} u(t)=\frac{A(s)}{A(s)} y(t)-\frac{B(s)}{A(s)} u(t) .
$$

If we define a low pass filter $L(s)=\frac{1}{A(s)}$ and substitute

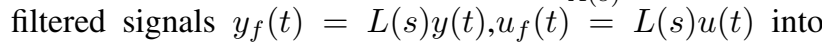
Eq. (3), then

$$
\begin{gathered}
\qquad(t)=A(s) y_{f}(t)-B(s) u_{f}(t) \\
=y_{f}^{\left(n_{a}\right)}(t)+a_{1} y_{f}^{\left(n_{a}-1\right)}(t)+\ldots+a_{n_{a}} y_{f}(t) \\
\quad-b_{1} u_{f}^{\left(n_{b}-1\right)}(t)-\ldots-u_{n_{b}} u_{f}(t), \\
\text { where } \begin{cases}y_{f}^{(i)}(t)=\mathcal{L}^{-1}\left\{\frac{s^{i}}{A(s)}\right\} * y(t), & i=1, \ldots, n_{a} \\
u_{f}^{(j)}(t)=\mathcal{L}^{-1}\left\{\frac{s^{j}}{A(s)}\right\} * u(t), & j=1, \ldots, n_{b} .\end{cases}
\end{gathered}
$$

Here, the symbol $\mathcal{L}^{-1}$ and $*$ denote the inverse Laplace transform and convolution product respectively. By evaluating Eq. (4) at sampling instants $\left\{t_{k}\right\}_{k=1}^{N}$, we have $N$ equations in $n_{a}+n_{b}$ variables, which are the model parameters $\theta=\left(a_{1}, a_{2}, . ., a_{n_{a}}, b_{1}, \ldots, b_{n_{b}}\right)$. If $N>n_{a}+n_{b}$, a unique solution of the model parameters can be found by a least mean square method. A detailed investigation of the SRIVC technique is documented in [11]. The implementation of the SRIVC method is available in CONTSID toolbox for Matlab [12].

\section{TRANSFORMER WINDING MODEL IDENTIFICATION}

\section{A. Data collection}

A test arrangement, as illustrated in Fig. 3, shows a process of acquiring data for identifying the impedance $Z_{1 a 1 b}(s)$ between two terminals $1 a$ and $1 b$ (Fig. 3(a)), and for estimating the transfer function $H_{12}(s)$ from port 1 to port 2 of the three-port network T (Fig. 3(b)). $V_{R B S}(t)$ is a general random binary signal having a flat spectrum over the frequency range of interest. In the case of impedance estimation, as presented in Fig. 3(a), a sensing resistor $R_{s}$ is purposely inserted into the test circuit to capture the current information. The excitation voltage $V_{R B S}(t)$ and the response voltage $V_{0}(t)$ are collected using data acquisition cards.

Although $V_{R B S}(t)$ and $V_{0}(t)$ generally serve as input and output data for system identification respectively, their role 

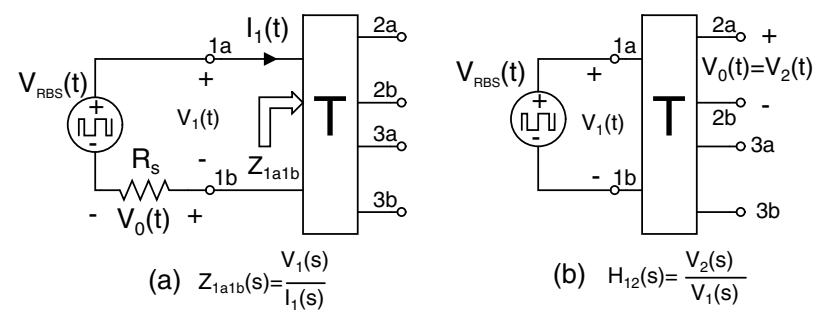

Fig. 3. Test circuits to collect time-series data for estimation of (a) an impedance and (b) a transfer function.

can be altered depending on the objective of the system under test. For example, referring to Fig. 3(a), a causal impedance $Z_{1 a 1 b}(s)$ can be either calculated indirectly from the identification result with input $V_{R B S}(t)$ and output $V_{0}(t)$ (according to Eq. (5)), or extracted directly by considering $V_{0}(t)$ as the input and $R_{s}\left(V_{R B S}(t)-V_{0}(t)\right)$ as the output (see Eq. (6)).

$$
\begin{aligned}
G(s) & =\frac{V_{0}(s)}{V_{R B S}(s)}=\frac{R_{s}}{R_{s}+Z_{1 a 1 b}(s)} \\
Z_{1 a 1 b}(s) & =\frac{V_{1}(s)}{I_{1}(s)}=\frac{R_{s}\left(V_{R B S}(s)-V_{0}(s)\right)}{V_{0}(s)}
\end{aligned}
$$

The difficulties of the time domain method arise when the magnitude response of the system varies significantly over a wide frequency range and can not be sufficiently resolved by the data acquisition card. The round-off error occurring will distort the estimated result regardless of the identification techniques used. Two options, consisting of increasing the resolution of each sample (more expensive measurement devices) and/or narrowing the observing frequency range, can be used to reduce the round-off error. The latter approach can be easily applied to impedance estimation by picking an appropriate value of $R_{s}$.

\section{B. Parameter extraction procedures}

A set of 8 different experiments is performed to derive all parameters for the winding model, as shown in Fig. 1. To clarify the measurement and identification procedure, a fixed template for each experiment, described below, is introduced. Noted that the transformer terminals are named in accordance with the three-port network in section III-A.

i) Test arrangement specification: presents which structure in Fig. 3 is followed to collect the time-series data. For example, $M Z_{2 a 3 a}$ indicates that using the set up in Fig. 3(a) but terminal $1 a$ and $1 b$ are replaced with $2 a$ and $3 a$. While $M H_{23}$ implies that the test in Fig. 3(b) is applied with the excitation signal $V_{R B S}(t)$ injected into port 2 and the response voltage $V_{0}(t)$ captured at port 3.

ii) Short circuit specification: describes the short circuit connection between transformer terminals. For example, $V_{3 a 3 b}=0$ means that making a short circuit connection between terminal $3 a$ and $3 b$. Noted that any terminals, which are not mentioned in this item, will be left in the default configuration as depicted in Fig. 1.

iii) The identification objective: denotes the transfer function and/or model parameters obtained from measured data.

Experiment 1:

i) $M Z_{1 a 1 b}$

ii) No short circuit connection

iii) $\frac{Z_{c m}(s) Z_{m}(s)}{Z_{c m}(s)+Z_{m}(s)}, R_{m}, L_{m}, C_{m}$

Experiment 2:

i) $M Z_{1 a 1 b}$

ii) $V_{2 a 2 b}=0$

iii) $Z_{1}(s)+\frac{Z_{2}(s) Z_{m}(s)}{Z_{2}(s)+n_{2}^{2} Z_{m}(s)}$

Experiment 3:

i) $\mathrm{MH}_{13}$

ii) $V_{2 a 2 b}=0$

iii) $Z_{1}(s), Z_{2}(s)$

Experiment 4:

i) $M Z_{1 a 1 b}$

ii) $V_{3 a 3 b}=0$

iii) $Z_{1}(s)+\frac{Z_{3}(s) Z_{m}(s)}{Z_{3}(s)+n_{3}^{2} Z_{m}(s)}$

\section{Experiment 5:}
i) $\mathrm{MH}_{12}$
ii) $V_{3 a 3 b}=0$
iii) $Z_{1}(s), Z_{3}(s)$

Experiment 6:
i) $M Z_{1 a 2 a}$
ii) $V_{1 a 1 b}=0, V_{2 a 2 b}=0, V_{3 a 3 b}=0, V_{2 a 3 a}=0$
iii) $Z_{C_{12}+C_{13}}(s), C_{12}+C_{13}$

\section{Experiment 7:}
i) $M Z_{1 a 2 a}$
ii) $V_{1 a 1 b}=0, V_{2 a 2 b}=0, V_{3 a 3 b}=0, V_{1 a 3 a}=0$
iii) $Z_{C_{12}+C_{23}}(s), C_{12}+C_{23}$

\section{Experiment 8:}
i) $M Z_{2 a 3 a}$
ii) $V_{1 a 1 b}=0, V_{2 a 2 b}=0, V_{3 a 3 b}=0, V_{1 a 2 a}=0$
iii) $Z_{C_{23}+C_{13}}(s), C_{23}+C_{13}$

We define the voltage transform ratios $n_{2}=\frac{N_{2}}{N_{1}}$ and $n_{3}=\frac{N_{3}}{N_{1}}$, where $N_{1}, N_{2}$ and $N_{3}$ are the number of turns in first, second and third windings. In order to explain how to obtain the model parameters from each experiment, the following assumptions are made:

- The inter-winding capacitances $C_{12}, C_{23}$ and $C_{13}$ can be identified separately from the rest of the transformer model.

- The impedance $Z_{m}(s)$ is typically much larger than $Z_{1}(s)$, hence we can neglect $Z_{1}(s)$ in Exp. 1 .

- The impedance of $C_{m}$, say $Z_{c m}(s)$, is much higher than $Z_{1}(s), Z_{2}(s)$ and $Z_{3}(s)$, therefore $Z_{c m}(s)$ is neglected in Exps. 2, 3, 4 and 5. 
The derivation of the impedance $Z_{1}(s), Z_{2}(s)$ in Exp. 3 requires an extra processing step. The relation between the signals $\left(V_{1}(t)\right.$ and $\left.V_{3}(t)\right)$, captured in Exp. 3, is given by

$$
V_{3}(s)=\frac{n_{3} \frac{Z_{2}(s) Z_{m}(s)}{Z_{2}(s)+n_{2}^{2} Z_{m}(s)}}{Z_{1}(s)+\frac{Z_{2}(s) Z_{m}(s)}{Z_{2}(s)+n_{2}^{2} Z_{m}(s)}} V_{1}(s) .
$$

Dividing both side of Eq. (7) by $\frac{Z_{2}(s) Z_{m}(s)}{Z_{2}(s)+n_{2}^{2} Z_{m}(s)}$, we can rearrange Eq. (7) into the form

$$
\frac{1}{Z_{2}(s)}=\frac{n_{3}\left(\hat{I}_{1}(s)-\hat{I}_{m}(s)\right)}{n_{2}^{2} V_{3}(s)}
$$

where

$$
\begin{gathered}
\hat{I}_{1}(s)=\frac{V_{1}(s)}{Z_{1}(s)+\frac{Z_{2}(s) Z_{m}(s)}{Z_{2}(s)+n_{2}^{2} Z_{m}(s)},} \\
\hat{I}_{m}(s)=\frac{V_{3}(s)}{n_{3} Z_{m}(s)} .
\end{gathered}
$$

where $\hat{I}_{1}(t)$ and $\hat{I}_{m}(t)$ denote the estimate of the primary current $I_{1}(t)$ and the magnetizing current $I_{m}(t)$ respectively. Since $Z_{1}(s)+\frac{Z_{2}(s) Z_{m}(s)}{Z_{2}(s)+n_{2}^{2} Z_{m}(s)}$ and $Z_{m}(s)$ are available from Exps. 1 and 2, we can easily calculate $\hat{I}_{1}(t)$ and $\hat{I}_{m}(t)$ from $V_{1}(t)$ and $V_{3}(t)$ via Eqs. (9) and (10). Using signals $n_{2}^{2} V_{3}(t)$ and $n_{3}\left(\hat{I}_{1}(t)-\hat{I}_{m}(t)\right)$ as data for system identification, one can directly obtain $Z_{2}(s)$ (according to Eq. (8)). In the case of $Z_{1}(s)$ estimation, Eq. (7) is modified as,

$$
\frac{1}{Z_{1}(s)}=\frac{\hat{I}_{1}(s)}{V_{1}(s)-\frac{V_{3}(s)}{n_{3}}} .
$$

where $\hat{I}_{1}(s)$ is given by Eq. (9). As can be seen in Eq. (11), $\hat{I}_{1}(t)$ (can be computed from $V_{1}(t)$ using Eq. (9)) and $V_{1}(t)-\frac{V_{3}(t)}{n_{3}}$ are enough to extract the impedance $Z_{1}(s)$. It should be noted that the extraction of the impedance $Z_{1}(s)$ and $Z_{3}(s)$ in Exp. 5 can be carried out in the similar way as implemented in Exp. 3. Since the impedance $Z_{1}(s)$ can be obtained from either Exps. 3 or 5, the similarity of the two results will act as a validation for the estimation technique.

In addition to the input-output data, a properly chosen order for the transfer function is a prerequisite for a system identification procedure. Fortunately, the transfer function order can be computed based on the transfer function order of the impedance that we want to determine in each experiment. For the frequency independent parameters, say $L_{m}, R_{m}, C_{m}, C_{12}, C_{23}$ and $C_{13}$, their corresponding impedances have a fixed order. By contrast, the impedance $Z_{1}(s), Z_{2}(s)$ and $Z_{3}(s)$ of the frequency dependent parameters defined in Eq. (12) have an undefined order.

$$
Z_{i}(s)=\frac{b_{i 1} s^{n_{i b}-1}+b_{i 2} s^{n_{i b}-2}+\ldots+b_{i n_{i b}}}{s^{n_{i a}}+a_{i 1} s^{n_{i a}-1}+\ldots+a_{i n_{i a}}}, i=\{1,2,3\}
$$

However, the relation between $\left\{n_{i b}\right\}_{i=1}^{3}$ and $\left\{n_{i a}\right\}_{i=1}^{3}$ in Eq. (12) has to be followed,

$$
n_{i b}=n_{i a}+2, i=\{1,2,3\} .
$$

The condition in Eq. (13) is introduced to limit the orders so that an obtained $\left\{Z_{i}(s)\right\}_{i=1}^{3}$ can be represented by a Foster's network and, hence, can be readily used in electrical simulators [7].

\section{EXPERIMENTAL RESULTS}

\section{A. Winding model determination}

The procedure, described in section III, is applied to a 3-winding transformer designed for a flyback converter application with a specification of an output power $90 \mathrm{~W}$, a switching frequency $100 \mathrm{KHz}$, an input voltage range $V_{i}=113-373 \mathrm{~V}$ and an output voltage $V_{o}=32 \mathrm{~V}$. The number of turns of the first, second and third windings are 46, 10 and 6 respectively. A ferrite core (EE type, Ferroxcube 3C95) with an air gap length of approximately $0.1 \mathrm{~mm}$ is required to provide an expected inductance of $800 \mu \mathrm{H}$. A signal generator circuit is designed and implemented to provide a symmetrical random binary voltage $V_{R B S}(t)$, having an adjustable amplitude $0 \mathrm{~V}-2 \mathrm{~V}$, a clock of $5 \mathrm{MHz}$ and a flat spectrum from $100 \mathrm{~Hz}$ to $5 \mathrm{MHz}$. The inputoutput data is acquired by an Agilent digital oscilloscope (DSO6054A) with a preset sampling rate of $50 \mathrm{MHz}$ and 12 bits for each sample. A sensing resistor of $465.5 \Omega$ is chosen for Exps. 1, 6, 7 and 8, while Exps. 2 and 4 use a resistor of value $R_{s}=6.84 \Omega$. The transfer function orders of the impedances $Z_{1}(s), Z_{2}(s)$ and $Z_{3}(s)$ are selected $\left\{n_{i b}\right\}_{i=1}^{3}=3$ and $\left\{n_{i a}\right\}_{i=1}^{3}=1$ in order to maximize the fit between the measured and simulated data.

The estimated parameters of the winding model are summarized in Table I, where the impedances $Z_{1}(s)$ obtained from both Exps. 3 and 5 are all included for comparison. As can be seen in Table I, the two independent estimations of $Z_{1}(s)$ are very close. This effectively confirms the accuracy of our estimation approach.

TABLE I

WINDING MODEL PARAMETERS

\begin{tabular}{|c|c|}
\hline Component & SRIVC Algorithm \\
\hline$L_{m}$ & $795.24 \mu H$ \\
\hline$R_{m}$ & $87.153 k \Omega$ \\
\hline$C_{m}$ & $8.864 p F$ \\
\hline$C_{12}$ & $7.8907 p F$ \\
\hline$C_{13}$ & $123.95 p F$ \\
\hline$C_{23}$ & $62.77 p F$ \\
\hline$n_{2}$ & 0.2174 \\
\hline$n_{3}$ & 0.1304 \\
\hline$Z_{1}(s)$ & $0.327+1.012 \cdot 10^{-6} s+\frac{0.908 s}{s+4.29 \cdot 10^{6}}$ from Exp. 3 \\
& $0.326+1.009 \cdot 10^{-6} s+\frac{0.834 s}{s+4.37 \cdot 10^{6}}$ from Exp. 5 \\
\hline$Z_{2}(s)$ & $0.0408+1.224 \cdot 10^{-7} s+\frac{0.125 s}{s+5.924 \cdot 10^{6}}$ \\
\hline$Z_{3}(s)$ & $0.0415+7.399 \cdot 10^{-8} s+\frac{0.0454 s}{s+3.233 \cdot 10^{6}}$ \\
\hline
\end{tabular}




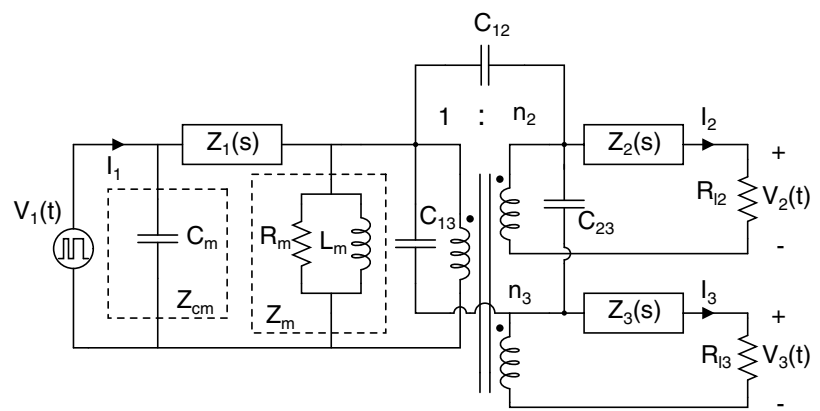

Fig. 4. A circuit structure for validating the transformer winding model in time domain.
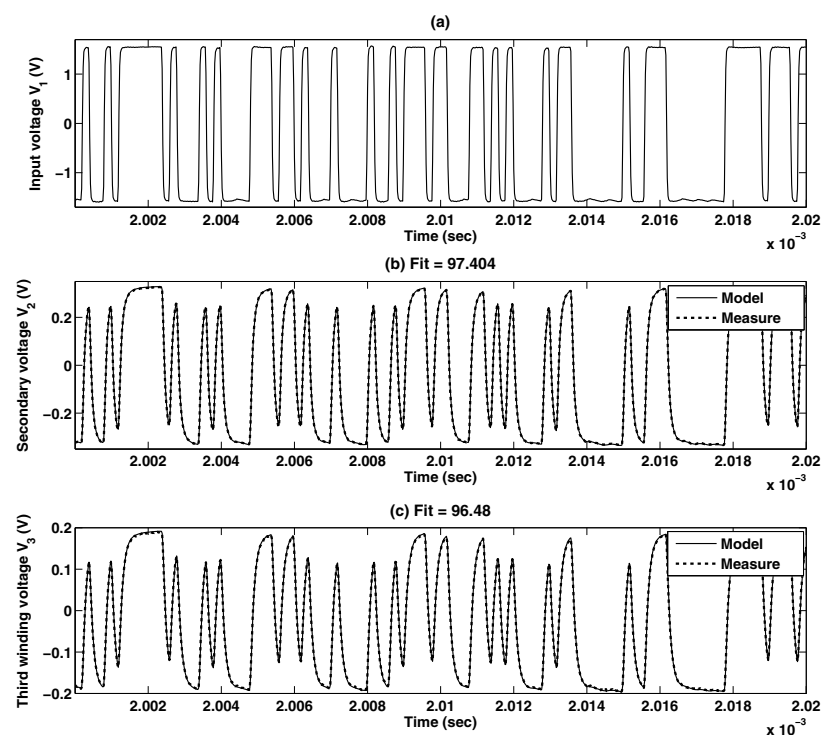

Fig. 5. Measured and simulated results obtained from the time domain model validation scheme in Fig. 4

\section{B. Model verification}

Both time and frequency domain tests are required to examine the accuracy of the obtained winding model. The time domain test circuit, as sketched in Fig. 4, is both experimentally performed and simulated in Simulink. A binary random voltage $V_{1}(t)$, as plotted in Fig. 5(a), is injected to the primary side of the transformer. Two load resistors $R_{l 2}=3.034 \Omega$ and $R_{l 3}=1.056 \Omega$ are chosen. The secondary and third winding voltage, which are returned from the experiment and model simulation, are compared in Fig. 5(b) and Fig. 5(c) respectively. The similarity and good fit between the simulated and measured output voltages strongly supports the time domain approach to determine the transformer model. The comparison with different load resistors, not presented here due to space limitation, also shows a good agreement between modelling and experimental results.
A test in the frequency domain is performed using a HP4194A Impedance/Gain-Phase Analyzer. The primary impedance of the transformer is measured between $500 \mathrm{~Hz}$ and $15 \mathrm{MHz}$ in three different circuit configurations as presented in Table II.

TABLE II

FREQUENCY DOMAIN TEST CONFIGURATIONS.

\begin{tabular}{|c|c|}
\hline Test case & Winding configuration \\
\hline 1 & Open circuit both secondary and third winding \\
\hline 2 & $\begin{array}{r}\text { Open circuit secondary winding and } \\
\text { short circuit third winding }\end{array}$ \\
\hline 3 & $\begin{array}{c}\text { Short circuit secondary winding and } \\
\text { open circuit third winding }\end{array}$ \\
\hline
\end{tabular}

For comparison, the corresponding frequency behavior of the primary impedance is computed in Matlab based on the model parameters in Table I. The frequency responses resulting from the actual measurement and modelling computation at different secondary and third winding configurations (as mentioned in Table II) are described in Figs. 6, 7 and 8. In all the three frequency-domain test cases, the model shows very good performance in predicting the transformer response at frequencies larger than $10 \mathrm{~K} \mathrm{~Hz}$. However, the error between the measured and modelling results increases at lower frequencies (smaller than $10 \mathrm{~K} \mathrm{~Hz}$ ). The reason for the discrepancy at low frequencies is due to the limitation in the total data time which can be collected by the digital oscilloscope. An oscilloscope, that can store a longer time-series of data, is expected to bring a better transformer model, particularly at low frequencies.

\section{CONCLUSIONS}

In this study, we present a time domain approach to the identification of a frequency-dependent model for a 3-winding flyback transformer. The method requires only simple measurement equipment (such as a digital oscilloscope) to acquire the time domain data. Although the roundoff error occurring during the data collection phase limits the performance of the estimation procedure, the accuracy can be improved by employing a high resolution data acquisition card or focussing on a frequency or dynamic range of interest. The latter approach has been successfully applied to impedance estimation by picking an appropriate sensing resistors for each experiment. As demonstrating in the paper, the time domain approach can provide a winding model at least as accurate as obtained with the frequency response data. With a longer data time series record, a more accurate transformer model is obtained, particularly at low frequencies. The results in this paper are valid for relatively small signal variations and represent the dynamics of the transformer, particularly for high frequencies. Future work will extend the operational range of the model to include a nonlinear transformer core effect. 

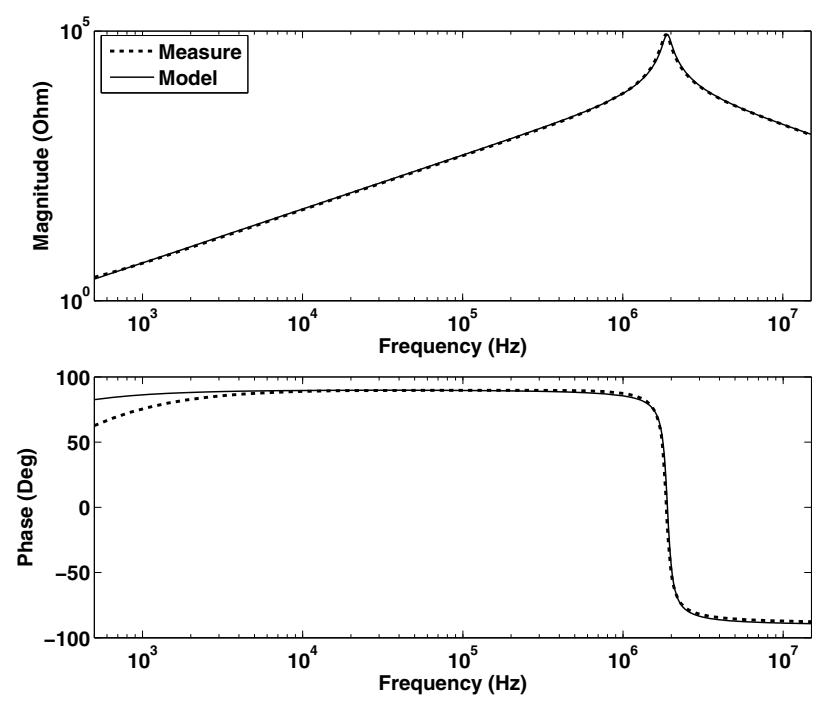

Fig. 6. Primary transformer impedance, with open circuit secondary and third windings.
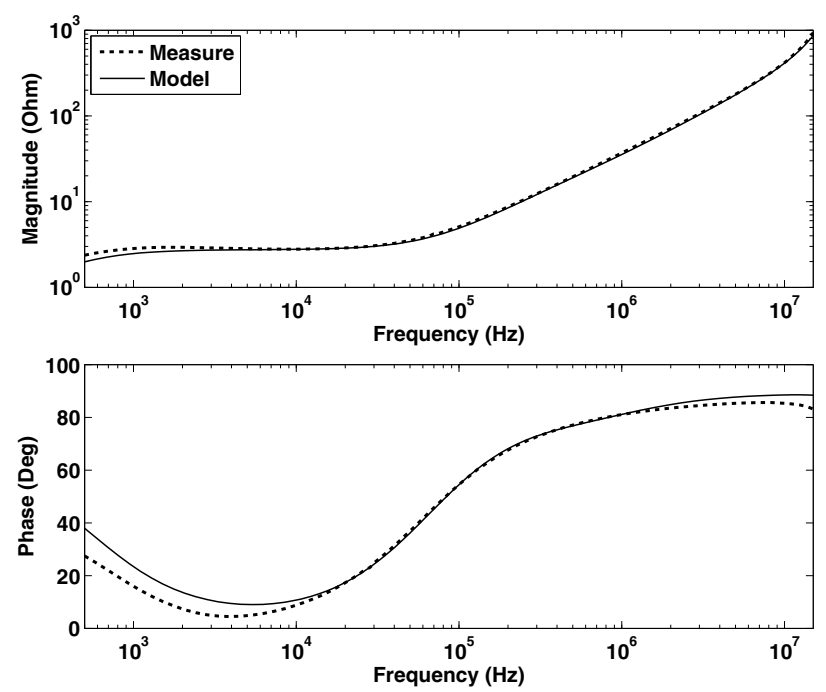

Fig. 7. Primary transformer impedance, with open circuit secondary and short circuit third winding.

\section{ACKNOWLEDGMENT}

The authors would like to thank Mr. James Kinsella from the Callan Institute (National University of Ireland, Maynooth) for support in setting up the measurement devices. Also special thanks to Dr. Philip Perry and Mr. Liam Carroll in University College Dublin for their valuable time in preparing the impedance analyzer.

\section{REFERENCES}

[1] P. Hsieh, L. Lee and K. Hsueh. (2010, Apr.). Driving higher levels of efficiency in lighting through primary side regulation. Fairchild Semiconductor, Taipei, Taiwan. [Online]. Available: http://www.ledinside.com/knowledge_20100429.
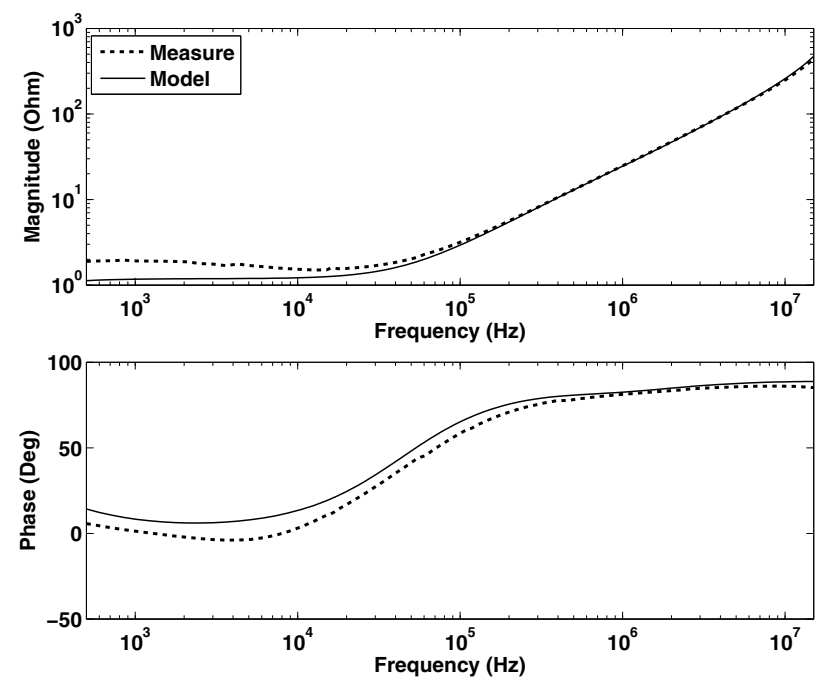

Fig. 8. Primary transformer impedance, with short circuit secondary and open circuit third winding.

[2] V. A. Niemela, H. A. Owen, and W. T. G., "Cross-coupledsecondaries model for multi-winding transformers with parameter values calculated from short-circuit impedance," in Proc. 21 st Annual IEEE Power Electronics Specialists Conference, San Antonio, TX, USA, pp. 822-830, Jun. 1990.

[3] A. Keyhani, H. Tsai, and S. Sebo, "Modelling and parameter estimation of power transformers for the study of high frequency system transients," in Proceedings of the 32nd Midwest Symposium on Circuits and Systems, Champaign, IL, pp. 258-264, Aug. 1989.

[4] J. Pleite, R. Prieto, R. Asensi, J. Cobos, and E. Olias, "Obtaining a frequency-dependent and distributed-effects model of magnetic components from actual measurements," IEEE Transactions on Magnetics, vol. 35, pp. 4490-4502, Nov. 1999.

[5] K. Ngo, S. Srinivas, and P. Nakmahachalasint, "Broadband extended cantilever model for magnetic component windings," IEEE Transactions on Power Electronics, vol. 16, pp. 551-557, Jul. 2001.

[6] P. Nakmahachalasint, K. Ngo, and L. Vu-Quoc, "A static hysteresis model for power ferrites," IEEE Transactions on Power Electronics, vol. 17, pp. 453-460, Jul. 2002.

[7] R. Asensi, J. Cobos, O. Garcia, R. Prieto, and J. Uceda, "A full procedure to model high frequency transformer windings," in Proc. 25th Annual IEEE Power Electronics Specialists Conference, Taipei, Taiwan, pp. 856-863, Jun. 1994.

[8] J. Bak-Jenson, B. Bak-Jenson, S. Mikkelsen, and C. Jensen, "Parametric identification in potential transformer modelling," IEEE Transactions on Power Delivery, vol. 7, pp. 70-76, Jan. 1992.

[9] S. Islam, K. Coates, and G. Ledwich, "Identification of high frequency transformer equivalent circuit using matlab from frequency domain data," in Proc. 32nd Annual IEEE Industry Applications Conference, New Orleans, Louisiana, USA, pp. 357-364, Oct. 1997.

[10] G. Rao and H. Unbehauen, "Identification of continuous-time systems," IEE Proceedings - Control Theory and Applications, vol. 153, no. 2, pp. 185-220, 2006.

[11] P. Young, J.W., H. Garnier, and M. Gilson, "An optimal instrumental variable approach for identifying hybrid continuous-time box-jenkins models," in 14th IFAC Symposium on System Identification, Newcastle, Australia, pp. 225-230, Mar. 2006.

[12] H. Garnier, M. Gilson and V. Laurain. (2009, Nov.). CONTSID toolbox for Matlab (Version 5.0). [Online]. Available: http://www.iris.cran.uhp-nancy.fr/contsid/Download.html. 